Recibido: 28-04-2016 Aceptado: 17-05-2016

Palabras clave: Neurociencias, neuromarketing, emoción, cognición. Keywords: neuroscience, neuromarketing, emotion, cognition

Alexander Chiu alexanderchw@gmail.com

$\mathrm{UPCH}$

Jessie Oré De Tomas jessie.ore@hotmail.com

UPCH

Rossana Stoddart ross.stoddart.landazuri@gmail.com $\mathrm{UPCH}$

Jorge Luis Linares Weilg jlinares@neurocienciasperu.org $\mathrm{UPCH}$

Carmen Estela Montenegro Serkovic carmen.montenegro@upch.pe $\mathrm{UPCH}$

Silvia Salinas nutriunife@yahoo.com UNIFE

Luis Aguilar luis.aguilar@upch.pe UPCH - UNIFE

\section{Avances en Neuromarketing: La influencia de la publicidad sobre "comida rapida" en el perfil emocional de los adultos}

\author{
Advances in neuro-marketing: The influence of \\ "fast-food" advertising in perception of taste and \\ adults' emotional profile
}

Alexander Chiu ', Jessie Oré1, Rossana Stoddart ${ }^{1,}$ Jorge Linares ', Estela Montenegro ${ }^{2}$ Silvia Salinas ${ }^{3}$, Luis Aguilar ${ }^{1,2,3}$

\section{RESUMEN.}

El marketing y la publicidad son disciplinas de carácter social enfocadas en el logro de objetivos de naturaleza comercial, esto se logra a través de la investigación de sus potenciales consumidores la cual sirve como insumo principal para la creación de estrategias de comunicación, entre otros aspectos empresariales, las cuales buscan persuadir a los consumidores hacia la adquisición de un determinado producto, servicio, idea o individuo, es por ello que la llamada "comida rápida", al igual que otros productos y servicios, se vale de ello para promover y vender sus productos a un público cuidadosamente seleccionado y estudiado.

La neurociencia es capaz de aportar en forma significativa con sus conocimientos, ya sea desde la fase de investigación inicial del consumidor hasta la creación y seguimiento de las estrategias de comunicación en base al conocimiento de patrones de comportamiento, siempre mencionando el aspecto ético de las acciones realizadas y los objetivos buscados por las empresas. Los objetivos empresariales y los científicos podrían encontrarse en una gran disputa considerando la naturaleza de cada ámbito y los fines que persigue, ya que en muchas ocasiones los ejecutivos de una empresa buscan básicamente la rentabilidad de sus instituciones mientras que los cientificos buscan la mayor objetividad en el uso de la información y del impacto que pueda causar el uso de un producto en la población, por ello el aspecto ético seguirá siendo un tema pendiente.

Los seres humanos se comportan de maneras muchas veces impredecible aunque en algunas ocasiones es posible detectar patrones de comportamiento que podría apoyar al establecimiento de estrategias capaces de lograr las metas establecidas por las empresas. Estos patrones podrían evidenciarse a nivel biológico, genético, psicológicas y/o social por lo cual la neurociencia tiene mucho de decir al respecto. En el presente estudio realizado en 
base a productos de "comida rápida" y la comunicación empleada para presentarlos, podemos notar una diferencia en el impacto en la decisión determinado por la estrategia de comunicación la cual busca impactar en el consumidor a nivel emocional. La publicidad busca impactar a su público objetivo desde una perspectiva creativa para llamar la atención en referencia al producto o servicio ofrecido haciéndolo atractivo, para ello utiliza información relevante sobre cada variable relacionada tanto al producto como de los consumidores, obteniendo así un mayor conocimiento del consumidor, $\mathrm{y}$ apelando principalmente al aspecto emocional, y es en este punto donde se unen las disciplinas comerciales con el apoyo del conocimiento neurocientífico para establecer las mejores estrategias de comunicación para desarrollar campañas publicitarias a través de conocimiento del consumidor

ABSTRACT. Marketing and advertising are social disciplines focused on achieving objectives of a commercial nature, this is achieved through research of your potential consumers it serves as the main input for the creation of communication strategies, among other business aspects, which seek it persuade consumers to purchase a particular product, service, idea or individual, which is why the "fast food", like other products and services, uses it to promote and sell their products to a carefully selected and studied public. Neuroscience is able to contribute significantly to their knowledge, either from the initial phase of consumer research to the creation and monitoring of communication strategies based on knowledge of behavior patterns, always mentioning the ethical aspect of the actions undertaken and the objectives sought by companies. Business and scientific objectives could find themselves in a dispute considering the nature of each area and the aims, since in many cases the executives of a company basically seeking the profitability of their institutions while scientists seek greater objectivity in the use of information and the impact caused by the use of a product in the population, so the ethical aspect remains an unresolved issue. Humans behave in ways often unpredictable but sometimes you can detect patterns of behavior that could support the establishment of strategies capable of achieving the goals set by the companies. These patterns may be evident to biological, genetic, psychological and / or social level which neuroscience has much to say about it. In the present study based on products of "fast food" and used to present communication, we can notice a difference in the impact on the particular decision communication strategy which seeks to impact the consumer on an emotional level. Advertising seeks to impact your target audience from a creative perspective to draw attention in reference to the product or service making it attractive, for it uses relevant information about each variable related to both the product and consumers, thus obtaining a greater consumer awareness and appealing primarily to the emotional aspect, and it is at this point that the trade disciplines supported by neuroscientific knowledge together to establish better communication strategies to develop advertising campaigns through consumer awareness. 


\section{Introducción}

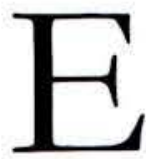

1 sobrepeso y la obesidad son considerados el quinto riesgo de mortalidad a nivel mundial según la Organización Mundial de la Salud (OMS) [1], dado que son causantes de enfermedades cardiovasculares, cáncer, enfermedades respiratorias crónicas, diabetes, entre otros. Es colinda al 2010, año que se estimaron más de 42 millones de menores de cinco años obesos o con sobrepeso en todo el mundo. Dicha tendencia no es ajena al Perú donde según el INEI la tasa de sobrepeso y obesidad infantil en el sector urbano es de $18 \%$ y $12 \%$, respectivamente $[2,7]$.

Los medios de comunicación, principalmente la televisión, son considerados uno de los principales medios de difusión, causantes del incremento de consumo de comida rapida, dando como respuesta el aumento de la obesidad en el mundo, tanto porque su consumo excesivo conduce al sedentarismo y a la ingesta de alimentos ricos en azúcares y grasas, como por la cantidad de publicidad de comida rapida que se publicita [3].

Del mismo modo, el ritmo de vida urbano, acelerado y estresante lleva a la población mundial a consumir productos que estén considerados más a la mano, entre ellos, se encuentran los de comida rápida. Este tipo de comidas contiene grasas, carbohidratos, preservantes, colorantes y carece control de muchos tipos de vitaminas, proteínas y demás nutrientes que son vitales dentro de la alimentación.

Frente a esta situación, el presente artículo plantea determinar el nivel de influencia de la publicidad de comida rápida en la toma de decisiones en individuos que ya cuentan con un aprendizaje previo, decisiones tomadas con un mayor nivel de planificación.

McDonald's es una de las cadena más grandes de comida rápida en el mundo. Esta marca se ha convertido en un símbolo de la globalización y el predominio de la cultura americana. Dicha marca no necesita de mucha actividad publicitaria en la actualidad ya que tiene un posicionamiento establecido a nivel global. Se desarrolla en cada país y se adapta a toda cultura y posee un mercado meta muy preciso, incluyendo a niños entre 7 a 14 años. Sin embargo, existen también productos para adultos o familias en donde promueve campañas especializadas en responsabilidad social.

Por otro lado, en el panorama local existe la empresa Bembos fundada el 11 de Junio de 1988 en Miraflores llegando a posicionarse en la actualidad como la hamburguesa de bandera, con orientación a adultos jóvenes, a diferencia de las marcas estadounidenses de comida rápida cuyo público objetivo principal son los niños. Su atributo de diferenciación no radica sólo en su grupo objetivo sino en la posibilidad de ofrecer una hamburguesa de proporciones más grandes. Actualmente cuenta con 33 establecimientos en Lima y presencia en el extranjero como Panamá, India (utilizando carne de soya), entre otros.

Finalmente, uno de los principales acompañantes de la comida rápida es la marca Coca- Cola. Se originó el 8 de mayo de 1886 y cosechando hasta el día de hoy éxitos rotundos al nivel mundial. Desde sus inicios, se destacaron sus cualidades (calidad y sabor) ganándose así un espacio en el mercado siendo considerado en el día de hoy como una de las mejores marcas mundiales. Su principal concepto publicitario es vender "felicidad" a toda la persona que lo consume teniendo un impacto principal en los niños y de manera secundaria en los adultos. A la fecha cuenta con presencia en más de 155 países alrededor del mundo

\section{Hipótesis:}

Si, la publicidad de comida rápida influye en el lado emocional del cerebro, entonces los individuos tenderán a a elegir en funcion a la percepcion de un mejor sabor y las marcas más 
afines a ellos o cuya historia detrás sea más emotiva en el lado positivo.

\section{Fundamentación teórica}

La publicidad es un esfuerzo pagado que utiliza los medios de comunicación masiva para persuadir a los consumidores al consumo de determinados productos y servicios. El impacto que tiene la publicidad, sobre todo televisiva, es considerable: una encuesta realizada por el Concortv a niños y adolescentes peruanos [4] determinó que el $96 \%$ recuerda haber visto spots publicitarios de gaseosas, helados, golosinas y comida rápida y las principales acciones tomadas fueron que "compraría el producto en algún momento" ( $29 \%)$, "lo compró" ( $26 \%)$, "no me provocó comprarlo" $(25 \%)$ y "le pidió a sus padres que lo compren" (20\%).

Por otro lado, según los últimos estudios del sector Salud, el $62.3 \%$ de la población, entre 30 y 59 años sufre de sobrepeso u obesidad. En otras palabras, tres de cada cinco adultos tienen exceso de peso. Si no se adquiere pronto un estilo de vida saludable, en menos de tres décadas Perú tendrá uno de los más altos índices de la región con pacientes diabéticos, hipertensos, cardíacos, entre otras patologías [5].

En este contexto, es importante resaltar la $\tan$ comentada "Ley en contra la comida chatarra" (comida rápida). Ley de promoción de la alimentación saludable, que supervisa la publicidad relacionada con alimentos y bebidas no alcohólicas, tiene el objetivo es reducir las enfermedades vinculadas con el sobrepeso y obesidad, así como los males crónicos conocidas como no enfermedades no trasmisibles. Esta ley busca que los peruanos optemos por una decisión saludable al momento de elegir alimentos y es, básicamente, reguladora de la publicidad, empresas y medios de comunicación. Los publicistas y comunicadores, saben que la mejor publicidad es la emocional, lo cual ya no estará permitido en los productos considerados no saludables, es decir, la publicidad de esta clase de comida, deberá ser racional al $100 \%$.

Asimismo, la Asociación civil "El Poder del Consumidor" dió a conocer que México permanece como el país con mayor cantidad de anuncios de comida rápida por hora en programación infantil entre los países de la Organización para la Cooperación y Desarrollo (OCDE), ubicándose por encima de los Estados Unidos. [6]. Ocupa el segundo lugar en prevalencia de sobrepeso y Obesidad.[ 7]

El estudio de "El Poder del Consumidor" parte de un registro realizado entre marzo y abril del 2010, en Canal 5 de México, EN esta, se encontró que el promedio de anuncios de comida rápida por hora en programación infantil fue de 11.25. De acuerdo al estudio, el $46 \%$ de los anuncios utilizaron un regalo para provocar la demanda del producto y el $67.5 \%$ utilizó personajes para generar el vínculo afectivo del menor con la marca y el producto (8).

En el 2012, se analizaron 212 spots publicitarios de alimentos no saludables emitidos en la televisión peruana y se encontró que en el $40 \%$ participaban niños. Entre las técnicas publicitarias más usadas se encontraron el uso de narrativas fantasiosas $(31 \%)$, apelación al antojo $(19 \%)$ y la asociación con dibujos animados (11\%). El 13\% de los alimentos no saludables comunicaban que 'eran buenos para la salud' y solo el $6 \%$ promovía la actividad física $[7,8]$.

Es importante describir la importancia del crecimiento del cerebro humano en función a las edades. Debemos entender que el sistema neruivoso centralA partir de los 27 años, el cerebro sufre una disminución progresiva del volumen de sustancia gris y empieza a incrementarse la sustancia blanca $[7,9,11]$. Esta última debe su nombre a la presencia de fibras nerviosas mielinizadas que mejoran la velocidad de transmisión de los potenciales de acción [10]. 
Para el caso de los adultos, ya cuentan con una experiencia previa la cual es almacenada en el tálamo, las decisiones de compra se realizan activando la parte prefrontal del cerebro (se encuentra totalmente desarrollada) tomando una decisión más racional. Considera diferentes factores dentro de los cuales podemos identificar: precio, presentación, sabor, imagen de la marca, entre otros factores.

Existen varios factores determinantes en el desarrollo de la persona como consumidor. Cuando somos niños nos dejamos influenciar por la interacción del ambiente (entorno) y la cultura. Sin embargo, conforme vamos creciendo se consideran diferentes factores que se van adicionando para realizar una compra de un producto, como por ejemplo: factores personales (edad tiene el consumidor, ocupación, personalidad) factores psicológicos (motivación, percepción, aprendizaje previo) factores sociales, entre otros.

\section{Objetivo}

El objetivo fué determinar el grado de influencia de la marca y el storytelling en la percepción de sabor de los alimentos poco saludables.

\section{Metodología}

Se ha realizado un experimento de laboratorio a través de un cuestionario (test de producto) a 28 personas entre 25 y 30 años de edad que laboran en el distrito de San Isidro (Lima) en agosto de 2014.

Alimento poco saludable: específicamente una hamburguesa, una porción de papas fritas y una gaseosa. Se utilizó la escala de Likert para dos tipos de preguntas. En la primera, relacionada a la comparación del sabor entre una y otra, la opción 1 fue "definitivamente el producto 1 sabe mejor", la opción 3 "las dos saben igual" y la opción 5 "definitivamente el producto 2 sabe mejor". El segundo tipo de pregunta fue relacionada al sabor particular de cada producto, siendo la opción 1 "no me gusta para nada" y la 5 "me gusta mucho".

En la primera parte del experimento se solicitó a la persona que pruebe dos gaseosas que provenían de botellas con un etiquetado distinto (dos gaseosas distintas, marca 1 y marca 2 ) pese a que el contenido era el mismo (una gaseosa pura, marca 1). Luego de realizada la prueba de producto, respondieron a las preguntas.

En la segunda parte se les pedía que consumieran dos porciones de papas fritas provenientes de sobres y bolsas con etiquetados distintos (dos marcas distintas, marca 1 y marca 2 ) pese a que el contenido provenía del mismo proveedor (una sola marca, marca 1). Luego de realizada la prueba de producto, respondieron a las preguntas.

Finalmente, la tercera parte del experimento consistía en probar dos porciones de hamburguesa (Comida rapida), cuyas etiquetas mencionaban que la carne provenía de distintas fuentes, pese a que eran las mismas (hamburguesa clásica de marca 1). La etiqueta de la Carne 1 mencionaba que "provenía de reses que se alimentan de pastos frescos pero que pasan el $70 \%$ del tiempo en cubículos aislados debido a las limitaciones de espacio de los camales". La etiqueta de la Carne 2 mencionaba que "provenía de reses que se alimentan de pastos frescos y que se mantienen el $70 \%$ del tiempo en campos extensos al aire libre". Luego de realizada la prueba de producto, respondieron a las preguntas.

\section{Resultados y Discusión}

El resultado obtenido en el test de marcas distintas es que el $64 \%$ de los participantes declararon que marca 1 sabía ligera o definitivamente mejor que marca 2 , mientras que el 36\% restante declaró que las dos sabían igual (opción correcta). Ningún participante declaró que marca 2 sabía mejor que marca 1 . La media 
Coca Cola tiene el mejor sabor

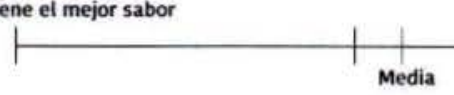

"Las dos saben igual"

Media
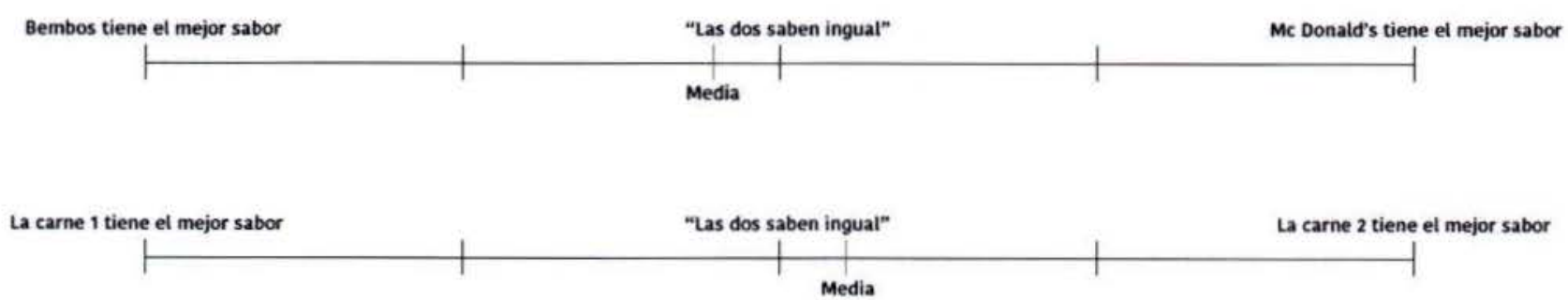

obtenida es 2.14 y la moda obtenida es 2 (marca 1, sabe ligeramente mejor que marca 2 de bebidas).

En las preguntas independientes sobre el sabor de bebidas, de cada producto, marca 1 obtuvo una media de 3.93 y moda de 4 ("me gusta"), mientras que marca 2 obtuvo una media de 3.64 y moda de 3 y 4 ("normal" y "me gusta").

El resultado obtenido en el test de comida rapida y papas fritas, marca 1 vs. Marca 2 es que el $36 \%$ de los participantes declararon que sabian igual (opción correcta), otro 36\% declaró que Marca 1 sabía mejor y el $28 \%$ restante que marca 2 sabía mejor. La media obtenida es 2.79 y la moda obtenida es 3 ("Las dos saben igual").

En las preguntas independientes sobre el sabor de cada producto, marca 1 obtuvo una media de 4.21 y moda de 4 ("me gusta"), mientras que marca 2 , obtuvo una media de 3.64 y moda de 4 ("me gusta").

El resultado obtenido en el test de Storytelling es que el $43 \%$ de los participantes declararon que las dos carnes sabían igual (opción correcta), el $36 \%$ que la carne 2 sabía mejor ("trato humano a las vacas") y el $21 \%$ restante que la carne 1 sabía mejor ("trato inhumano a las vacas"). La media obtenida es 3.21 y la moda obtenida es 3 ("Las dos saben igual').

En las preguntas independientes sobre el sabor de cada producto, la carne 1 marca 1, ("trato inhumano a las vacas") obtuvo una media de 4.07 y moda de 4 ("me gusta"), mientras que Marca 2, obtuvo una media de 4.29 y moda de 4 ("me gusta").

\section{VII.- Conclusiones}

De los datos obtenidos se puede concluir que las marcas de bebidas como Marca1 y comida marca 1, que son líderes en el mercado y están posicionadas a un público objetivo similar al que participó en el experimento tienen cierta influencia en la percepción de sabor. Esto puede obedecer a las prácticas publicitarias, estrategias de posicionamiento y branding que realizan a lo largo del tiempo y que pueden influenciar en el sistema de recompensa del cerebro, principalmente a través de la activación de vias dopaminergicas ubicados en centros precorticales como la amígdala, núcleo accumbens y glándula pituitaria.

Asimismo, se pude concluir que hay una ligera influencia del storytelling emocional en la percepción de sabor. Esto puede estar asociado a la activación de las neuronas espejo y el sistema límbico, específicamente en la amígdala a través de una mayor activación de las vias Gabaergicas y Glicinergicas.

\section{Bibliografía}

Chiu Werner; Alexander.2012. La obesidad infantil y la publicidad de alimentos no saludables en el Perú. (http://www.concortv.gob. pe/index.php/listado-de-articulos/866la-obesidad-infantil-y-la-publicidad-dealimentos-no-saludables-en-el-peru.html) (Fecha de consulta: 21 de junio de 2014) 
Consejo Consultivo de Radio y Televisión (CONCORTV). 2012. Encuesta a niños y adolescentes sobre la radio y televisión en el Perú. (http://www.concortv.gob.pe/index. php/investigaciones/encuestas/1041-2012encuesta-a-ninos-y-adolescentes-sobre-laradio-y-television-peruana.html) (Fecha de consulta: 21 de junio de 2014)

El Poder del Consumidor 2010. México, líder en publicidad chatarra.

(http://elpoderdelconsumidor.org/ saludnutricional/mexico-lider-enpublicidad-chatarra/ )(Fecha de consulta: 25 de agosto de 2014)

Gearhardt, Ashley N. 2013. Relation of obesity to neural activation in response to food commercials. (http://scan.oxfordjournals. org/content/early/2013/05/09/scan. nst059.full.pdf + html? sid $=90611000$ 12d8-4dec-89e0-c6dba9c5e489) (Fecha de consulta: 21 de junio de 2014)

González García, Silvia. 2003. Los niños como consumidores: impacto del estilo parental en el desarrollo de heurísticas para la toma de decisiones de bajo involucramiento. (http:// cladea.revistaleadership.com/doctoral/ silvia $\% 20$ gonzales.pdf) (Fecha de consulta: 21 de junio de 2014)

Kanai, Ryota \& REES, Geraint. 2011. The structural basis of inter-individual differences in human behaviour and cognition. Nature Reviews Neuroscience 12, 231-242. (http://www.nature.com/nrn/journal/ v12/n4/box/nrn3000 BX3.html) (Fecha de consulta: 21 de junio de 2014)

Roedder, Deborah. 1999. Consumer socialization of Children: a Retrospective Look at Twenty-Five Years of Research. (http:// www.carlsonschool.umn.edu/assets/72083. pdf) (Fecha de consulta: 21 de junio de 2014) Susuki, K..2010. Myelin: A Specialized Membrane for Cell Communication. Nature Education 3(9):59
[1] Cfr. OMS 2010

[2] Cfr. Rivadeneyra 2012

[3] Cfr. Pérez-Salgado, Rivera-Márquez y Ortiz-Hernández 2009

[4] Cfr. Concortv 2012.

[5] http://elcomercio.pe/lima/ciudad/tres-cada-cincoperuanos-tienen-sobrepeso-obesidad-noticia-1731163

[6] Cfr. El Poder del Consumidor 2010

7.- Escenario actual de la obesidad en México Antonio Barrera-Cruz,a Arturo Rodríguez-González,b Mario Antonio Molina-Ayalab. Instituto Mexicano del Seguro Social, Distrito Federal, México. 2012. Revisado el 4NOV2014 en http://www. medigraphic.com/pdfs/imss/im-2013/im133k.pdf

[8] Cfr. Chiu Werner 2012.

[9] Cfr. Kanai \& Rees 2011.

[10] Cfr. Susuki 2010.

11. Francisco Mora Envejecimiento cerebral . 2008. Spain 